\title{
Effectiveness of exercise in patients with osteoarthritis of hip or knee: nine months' follow up
}

\author{
M E van Baar, J Dekker, R A B Oostendorp, D Bijl, Th B Voorn, J W J Bijlsma
}

Netherlands Institute of Primary Health Care, Utrecht, The Netherlands

$M$ E van Baar

J Dekker

D Bijl

Institute of Research in Extramural

Medicine, Vrije

Universiteit,

Amsterdam, The

Netherlands

J Dekker

Dutch National

Institute of Allied

Health Professions,

Amersfoort, The

Netherlands

R A B Oostendorp

Department of

General Practice,

Utrecht University,

Utrecht, The

Netherlands

Th B Voorn

Department of

Rheumatology and

Immunology,

University Hospital,

Utrecht, The

Netherlands

J W J Bijlsma

Correspondence to:

Dr M E van Baar, Nivel, PO

Box $1568,3500 \mathrm{BN}$,

Utrecht, The Netherlands

v.d.m.dehaas@kub.nl

Accepted 23 April 2001

\begin{abstract}
Objective-To determine whether the effects of an exercise programme in patients with osteoarthritis of hip or knee are sustained at six and nine months' follow up. Methods-A randomised, single blind, clinical trial was conducted in a primary care setting. Patients with osteoarthritis of hip or knee (ACR criteria) were selected. Two intervention groups were compared. Both groups received treatment from their general practitioner, including patient education and drug treatment if necessary. The experimental group also received exercise treatment from a physiotherapist in primary care. The treatment period was 12 weeks, with an ensuing 24 week follow up. The main outcome measures were pain, drug use (non-steroidal anti-inflammatory drugs), and observed disability.
\end{abstract}

Results-201 patients were randomly allocated to the exercise or control group, and 183 patients completed the trial. At 24 weeks exercise treatment was associated with a small to moderate effect on pain during the past week (difference in change between the two groups $-11.5(95 \%$ CI -19.7 to -3.3$)$. At 36 weeks no differences were found between the groups.

Conclusions-Beneficial effects of exercise decline over time and finally disappear.

(Ann Rheum Dis 2001;60:1123-1130)

In treatment guidelines for osteoarthritis (OA) of the hip or knee the use of exercise has been advocated. ${ }^{12}$ In a recent update, exercise again is recommended as an important nonpharmacological treatment in patients with $\mathrm{OA}$ of the hip and knee. ${ }^{3}$

Exercise treatment in $\mathrm{OA}$ aims at reducing pain and disability. ${ }^{4}$ This is achieved through improvement of muscle strength, stability of joints, range of motion, and aerobic fitness. These functions are often impaired in patients with OA, presumably contributing to pain and disability. ${ }^{5}$ Improving these functions is assumed to reduce pain and disability. In addition, exercise aims directly at reducing disability - for example, through correction of the walking pattern. ${ }^{67}$

In a systematic review, based on 11 randomised controlled trials (RCTs), we concluded that there is evidence for the beneficial effects of exercise on pain, disability, and patient's global assessment. ${ }^{8}$ Since the publication of this review several new RCTs on exercise in patients with OA have been published. These newly published RCTs confirm this conclusion. ${ }^{9-13}$

Most randomised clinical trials, however, were confined to post-treatment results, ignoring residual effectiveness. Randomised controlled trials on the long term effects of exercise in patients with OA of the hip or knee are few. In four trials the residual effects of exercise were studied after completion of the programme. ${ }^{9}{ }^{13-15}$ Beneficial effects at one year were found in four trials, on pain, ${ }^{914}$ disability, ${ }^{14}$ and muscle strength. ${ }^{913}$ In one trial no beneficial effects could be shown. ${ }^{16}$ However, several methodological shortcomings hamper the interpretation of these results. These include the absence of an intention to treat analysis, ${ }^{13}$ a high loss to follow up, ${ }^{15}$ combined data presentation for different patient groups (OA and rheumatoid arthritis) ${ }^{14}$ or for different assessments, ${ }^{9}$ and a low power owing to small numbers of patients at randomisation. ${ }^{9}$

One trial studied the effectiveness of exercise consisting of a three month centre based phase followed by a 15 month home based phase. ${ }^{16}{ }^{17}$ The effects of exercise after completion of the exercise programmes were not studied. When data from follow ups at 3, 9, and 18 months were combined, beneficial effects were reported for pain, disability, muscle strength, and aerobic capacity. Separate follow up data were presented for a subset of outcome measures. These data indicated a maximal beneficial effect at three months (after completion of the supervised programme) for self reported disability and pain, and at nine months for walking speed. Most effects showed a slight decline afterwards, even when the home based exercise programme was continued.

In uncontrolled studies too, scarcely any attention has been paid to the residual effects of exercise in OA. Fisher et al reported beneficial follow up effects (eight and 12 months) of a four month muscle rehabilitation programme. ${ }^{18}$ Beneficial effects on pain and observed disability (including walking) remained at post-treatment levels, at both eight and 12 months. Beneficial effects on muscle strength and endurance had declined at 12 months, but remained significantly higher than baseline levels. In a case study, Marks presented effects of quadriceps exercises on muscle strength. ${ }^{19}$ After a six week supervised 
training period quadriceps torque had improved by $40 \%$, with a $10 \%$ reduction after a six week non-training period.

In conclusion, the residual effects of exercise in OA of the hip or knee after completion of treatment have been investigated in only a few studies. It remains unclear how long beneficial effects are sustained after completion of the exercise treatment.

We here report a follow up study to determine whether the effects of exercise in patients with OA of the hip or knee are sustained over time. The effects of exercise at 12 and 24 weeks after completion of a supervised exercise programme are presented. In addition, the prognostic value of patient characteristics for outcome after completion of treatment is explored. The short term effects of exercise in our trial have been reported in detail elsewhere. $^{20}$

\section{Patients and methods}

STUDY GROUP

Patients were selected by GPs in the period May 1994 to February 1996. The GPs were situated in four cities and surrounding villages in the eastern part of the Netherlands. Inclusion criteria for patients were $\mathrm{OA}$ of the hip or knee according to the clinical criteria of the American College of Rheumatology. ${ }^{21}{ }^{22}$ Exclusion criteria were another disease which might explain the complaints; complaints in fewer than 10 of 30 days; treatment for these complaints with exercise in the preceding six months; age under 40 or over 85 ; indication for hip or knee replacement; contraindication for exercise treatment; contraindications for analgesics or non-steroidal anti-inflammatory drugs (NSAIDs); and inability to understand the Dutch language. After having given oral consent, patients were registered and their names forwarded to the research team. Radiographs were obtained and evaluated by one radiologist using grading scales $(0-3)$ for individual radiographic features. ${ }^{23}$ All patients were visited and their eligibility was checked by a GP research fellow (DB). All eligible patients were asked to give written informed consent. Afterwards, patients were randomly allocated equally to either the exercise or the control group, using sequentially numbered, opaque, sealed envelopes containing the treatment assigned.

Patients were split into groups according to their pain in the past week (VAS 0-30 v 31-100 $\mathrm{mm}$ ) and location of OA (hip or knee) in order to achieve comparability in these prognostic factors.

We aimed at having 200 patients participating in the study, as this number of patients leads to a power of 0.80 to detect small to medium sized effects with an $\alpha$ of $0.05 .^{24}$

The study protocol was approved by the ethics committee of the Maastricht University Hospital (Maastricht, the Netherlands).

INTERVENTIONS

Two interventions were compared. The patients in the exercise group were given exercise treatment individually by a physiotherapist in primary care. In addition, their GP provided patient education (including a brochure) and drug treatment, if necessary. Treatment of the control group was restricted to that given by their GP, as described above (patient education and drugs, if necessary). The treatment period was 12 weeks, with 24 weeks of follow up.

Exercise treatment was given according to a written protocol ${ }^{25}$ and included exercises for muscle functions (strength and length), mobility, and coordination, and exercises for elementary movement abilities and locomotion abilities. Also, instructions for the adaptation of activities of daily living and home exercises were given. Content and intensity of treatment were described by treatment goals and corresponding exercises. Content, intensity, and frequency of treatment were tailored to the patient's needs. Depending on the physiotherapist's diagnostic findings, specific treatment goals with corresponding exercises were chosen. A number of sessions a week were prescribed, ranging from one to three times a week, depending on the pain level. A physiotherapy session in primary care lasted approximately 30 minutes. Exercise treatment could be discontinued within the 12 week period if, according to the physiotherapists, treatment goals had been achieved. Physiotherapists were trained to use the protocol.

A protocol was also used for the prescription of drugs. The GP prescribed preferably paracetamol; prescription of NSAIDs was restricted to naproxen, diclophenac natrium, and ibuprofen. The patient was instructed to use as few drugs as possible. The GP also provided a brochure for patient education, covering diagnosis, prognosis, advice about rest, daily activities and diet, the use of aids and medical treatment. No advice about exercise was included. GPs were instructed to minimise treatment in the follow up period. Physiotherapists and GPs recorded detailed information about the actual treatments on standardised forms, including any deviation from the protocol.

\section{OUTCOME ASSESSMENT}

Primary outcome measures

Primary outcome measures were pain, use of NSAIDs, and observed disability. Patients rated their pain in the past week on a visual analogue scale $(0 \mathrm{~mm}=$ no pain to $100 \mathrm{~mm}=$ very severe pain) in answer to the question: How severe was your pain during the past week? The amount of NSAIDs used was based on prescription data and a count of remaining drugs during evaluation sessions. Observed disability was determined by studying videos of the patients' performance of a series of standardised tasks, using an adaptation of the method described by Keefe et al. ${ }^{26}{ }^{27}$ The tasks included walking, sitting down, bending, and reclining. Movement times and quality of performance were both assessed. The interobserver reliability of this method is good. ${ }^{27-29} \mathrm{~A}$ total score was calculated based on five measures: $5 \mathrm{~m}$ walking time, stand to sit time, stand to recline time, and the levels of caution and rigidity during the performance of the 
tasks. Standardised scores ( $Z$ scores) of separate measurements were first calculated and then summed to obtain an overall score. To enhance comparability the resulting overall score was standardised to produce a score with a mean of zero and a standard deviation of one. $^{30}$ The internal consistency of the constructed overall score was good $(\alpha=0.84){ }^{31}$

\section{Secondary outcome measures}

A set of 13 secondary outcome measures were included in the trial, to study the working mechanisms of exercise treatment in OA. In this article, a subset of these secondary outcome measures is included: use of paracetamol assessed in the same way as the use of NSAIDs; global perceived effect as assessed by the patients themselves on an eight point scale ( 1 = vastly worsened; $8=$ completely recovered $)^{32}$; self reported disability with the IRGL questionnaire (Influence of Rheumatic disease on General health and Lifestyle) ${ }^{33}$; muscle strength measured with a hand held dynamometer $^{34}$; joint motion measured with a goniom$e^{e t e r}{ }^{35}$; and the level of physical activity according to the Zutphen Physical Activity Questionnaire (excluding sedentary hobbies). ${ }^{36}$ Overall scores were compiled for muscle strength of hip and knee and joint range of motion of hip and knee. The procedure was similar to data reduction in observed disability $\left(\right.$ mean $=0, \mathrm{SD}=1$, see van Baar et $\left.a l^{30}\right)$.

\section{Prognostic factors}

The following prognostic factors were studied:

- Demographic characteristics: age and sex

- Clinical features: location of OA of the hip (yes/no) and the knee (yes/no), OA of the hand according to Altman et $a l^{\beta^{7}}$ (yes/no), radiological OA as measured on grading scales for individual radiographic features (yes/no)(for details see van Baar et $a \beta^{0}$ ), obesity (body mass index $<30 / \geqslant 30^{38}$ ), duration of complaints and comorbidity (yes/no)

- Baseline levels of outcome measures: pain during the past week, drug use (NSAIDs, paracetamol), observed and self reported disability, muscle strength, and range of joint motion, both for hip and knee, and level of physical activity

- Pain coping strategies (six dimensions) as assessed with the Pain Coping Inventory. ${ }^{39}$ In addition, the extent to which patients believe physical activity to affect their pain was assessed using an adaptation for patients with OA of the Fear-Avoidance Beliefs Questionnaire ${ }^{40}$

- Psychological wellbeing: anxiety, depression, and cheerfulness as assessed with the IRGL questionnaire ${ }^{33}$

- Compliance with exercise as reported by both the patient and the physiotherapist. Patients were phoned by a research fellow $(\mathrm{MvB})$ at weeks 6 and 12 and asked, using standardised questions, whether they had done their home exercises as often as prescribed. Possible answers were: (almost) never, sometimes, regularly, often, and very often. This contact was not used to reinforce compliance with the exercise programme.
Physiotherapists, using a standardised form, were asked to estimate whether the patient complied with the home exercise instructions in the last four weeks of treatment. Possible answers were: certainly not, probably not, probably, and certainly. A patient was considered compliant (a) when the patient reported exercising (very) often or

(b) when the physiotherapist estimated that the patient had certainly exercised at home. Patients were evaluated by a "blinded" research assistant at baseline and at 12 weeks (the end of treatment), 24 weeks, and 36 weeks. The research assistants had been trained to perform the measurements in a standardised manner. The evaluations took place in local healthcare centres.

\section{STATISTICAL ANALYSIS}

Analyses were performed according to the intention to treat principle..$^{41}$ Patient data were analysed in the intervention groups to which they had initially been assigned. This included patients who had withdrawn and patients not treated according to the assigned treatment. In addition, a per-protocol analysis was performed, excluding patients with deviations from the treatment protocol and late ineligible patients.

To analyse the effects, change scores were calculated (follow up score minus baseline score). For drug use and global perceived effect, follow up scores were compared because no change scores could be calculated. The scores for global perceived effect and physical activity were dichotomised in order to study the number of patients who had improved. In the analysis, the change (or follow up) scores were compared between the interventions by analysis of covariance. Adjusted analyses were performed. The baseline level of each outcome measure was included to enhance the precision of the effect estimates. In addition, drug use and fear avoidance beliefs, the measures on which the groups differed at baseline (see "Results"), were included as covariates in order to control for baseline differences. Group differences and 95\% confidence intervals were calculated for all outcome measures. In addition, effect sizes were calculated by taking the difference between the change scores of the intervention groups and dividing it by the standard deviation of the change score of the total group. An effect size of 0.2 is considered to be a small beneficial effect of exercise, $0.5 \mathrm{a}$ medium effect, and 0.8 a large effect. ${ }^{24}$

The analysis for studying the prognostic value of patient characteristics for long term outcome of exercise was restricted to patients who had received exercise treatment. These patients were classified as (a) being stable or improved or $(b)$ having deteriorated, based on their results for the primary outcome measures during follow up (week 12 to week 36). Patients pain during the past week or observed disability was classified as stable or improved if their scores in week 36 were equal or lower than in week 12. Patients were classified as stable or improved for NSAIDs if they did not change drug use (or non-use) or if they had reduced or 


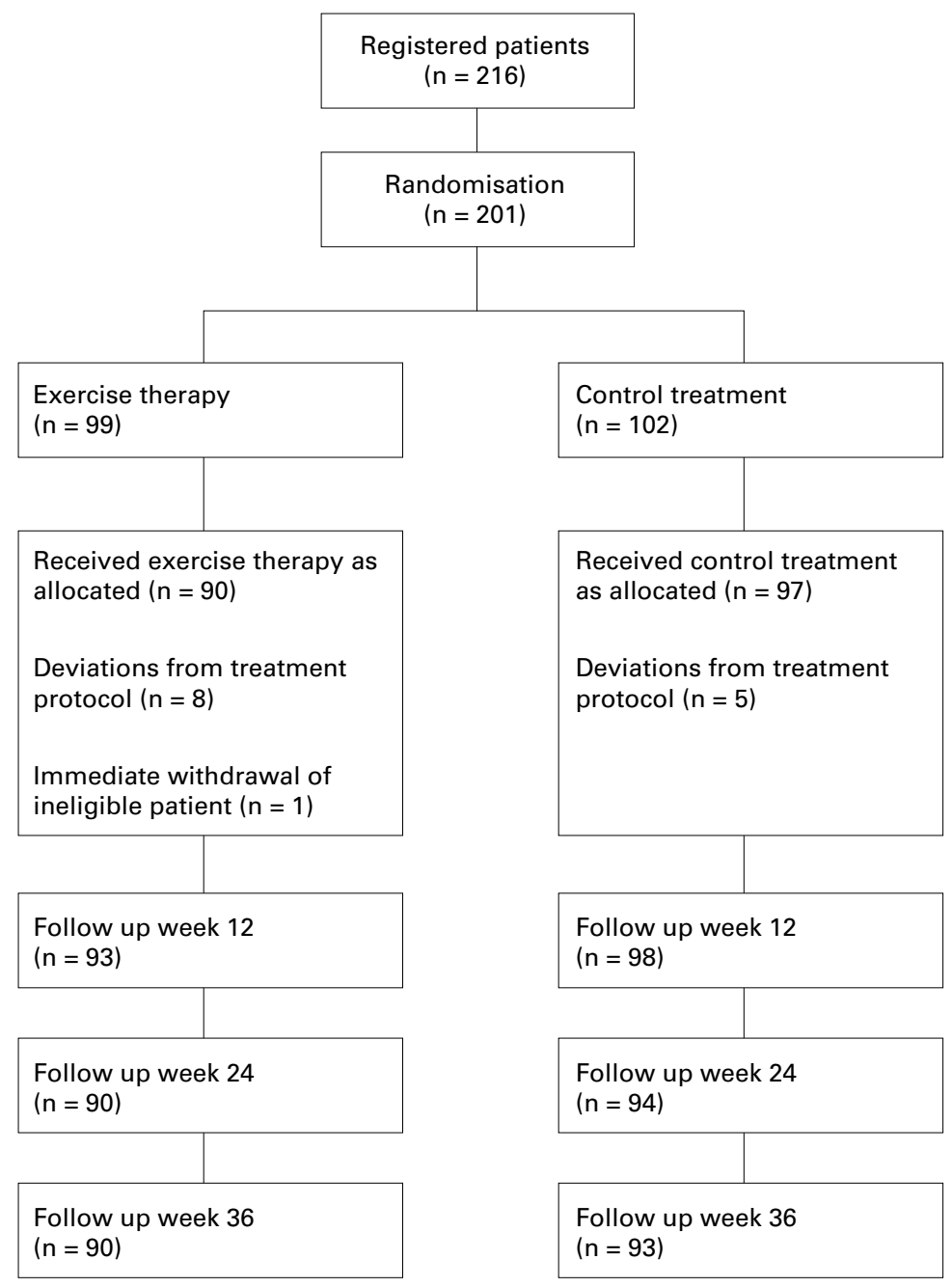

Figure 1 Participant flow and follow up.

Table 1 Comparability of intervention groups at baseline*. Results are given as number (\%) except where otherwise stated

\begin{tabular}{|c|c|c|}
\hline & $\begin{array}{l}\text { Exercise therapy } \\
(n=98)\end{array}$ & Control $(n=102)$ \\
\hline Sex (female) & $76(78)$ & $81(79)$ \\
\hline Age (years), mean (SD) & $68.3(8.4)$ & $67.7(9.2)$ \\
\hline Comorbidity & $63(64)$ & $62(62)$ \\
\hline \multicolumn{3}{|l|}{ Location of OA } \\
\hline Knee & $58(59)$ & $61(60)$ \\
\hline Hip & $36(37)$ & $35(34)$ \\
\hline Both & $4(4)$ & $6(6)$ \\
\hline \multicolumn{3}{|l|}{ Duration of complaints (years) } \\
\hline$\leqslant 0.5$ & $34(35)$ & $42(41)$ \\
\hline $0.5-\leqslant 1$ & $13(13)$ & $12(12)$ \\
\hline $1-\leqslant 5$ & $29(30)$ & $25(25)$ \\
\hline$>5$ & $22(22)$ & $23(23)$ \\
\hline \multicolumn{3}{|l|}{ Radiological OA (score $\geqslant 1$ ) } \\
\hline Joint space narrowing & $62(69)$ & $55(60)$ \\
\hline Osteophytes & $65(72)$ & $65(71)$ \\
\hline Previous medical treatment & $59(61)$ & $64(64)$ \\
\hline Pain in past week (VAS $+0-100)$, mean (SD) & $46.9(27.7)$ & $43.1(26.8)$ \\
\hline At assessment, $\dagger$ mean (SD) & $34.0(27.2)$ & $28.7(26.0)$ \\
\hline \multicolumn{3}{|l|}{ Drug use in past week (taking) } \\
\hline Paracetamol & $51(52)$ & $39(38)$ \\
\hline NSAIDst & $34(35)$ & $23(23)$ \\
\hline \multicolumn{3}{|l|}{ Observed disability } \\
\hline $5 \mathrm{~m}$ walking time $(\mathrm{s})$, median $(\mathrm{IQR} \dagger)$ & $4.9(4.3-6.0)$ & $5.0(4.3-6.0)$ \\
\hline Sit time $(\mathrm{s})$, median (IQR) & $3.4(2.9-4.1)$ & $3.4(2.9-4.0)$ \\
\hline Recline time (s), median (IQR) & $6.9(5.4-9.0)$ & $6.1(5.1-7.9)$ \\
\hline Guarding $(0-1)$, mean $(\mathrm{SD})$ & $0.58(0.48)$ & $0.53(0.50)$ \\
\hline Rigidity $(0-1)$, mean (SD) & $0.34(0.38)$ & $0.33(0.37)$ \\
\hline
\end{tabular}

${ }^{\star}$ Missing values: comorbidity ( 1 in exercise therapy, 2 in controls); duration of complaints ( 2 in exercise therapy, 6 in controls); radiological OA (7 in exercise therapy, 12 in controls); previous medical treatment ( 1 in exercise therapy, 2 in controls).

+VAS = visual analogue scale; NSAIDs = non-steroidal anti-inflammatory drugs; IQR = interquartile range. stopped using NSAIDs in the period week 12 to week 36. A $\chi^{2}$ test and $t$ test for independent samples were used to test for baseline differences or differences in compliance between these two groups.

The analyses were carried out using SPSS/ PC+ 5.0.

\section{Results}

PARTICIPANT FLOW

Figure 1 shows the patient flow and follow up between registration and week 36. In total 201 patients were included in the study, 99 patients were allocated to exercise therapy, 102 patients to the control treatment. One patient allocated to exercise treatment was excluded because of additional medical information (erythrocyte sedimentation rate $>45 \mathrm{~mm} / 1 \mathrm{st}$ h) obtained immediately after random allocation to the group.

The week 12 assessment was completed by 191 patients; 183 patients completed the trial up to 36 weeks. Reasons for withdrawal and loss to follow up were comorbidity $(n=8)$, loss of motivation $(n=6)$, family circumstances $(n=2)$, and adverse effects of exercise $(n=1)$. The number of patients withdrawing and lost to follow up was relatively low. In addition, numbers and reasons were equally divided across the intervention groups.

Table 1 presents the baseline characteristics in the two intervention groups. Patients were generally similar, and also with respect to OA and the contralateral leg (data not shown). There were two exceptions: patients allocated to the exercise treatment group reported a higher use of drugs in the seven days before participation in the study and scored higher on fear avoidance beliefs concerning physical activity.

\section{TREATMENT}

Patients receiving exercise treatment visited their GP 1.6 (SD 0.7) times in the 12 week treatment period, control patients $1.8(0.9)$ times $(\mathrm{p}=0.03)$. The GP prescribed drugs for $54 \%$ and $66 \%$ of the patients in the two groups, respectively $(\mathrm{p}>0.05)$.

In the exercise group the mean number of physical therapy sessions was 16.8 (SD 7.0). The median number of sessions was 14 (range 3-30). The median duration of treatment was 11 weeks; $38 \%$ of the patients received 12 weeks of treatment (for details, Baar et $a l^{19}$ ).

Compliance with home exercises was moderate to good: $66 \%$ of the patients reported exercising often or very often. The physical therapist estimated good compliance (that is, certainly did the home exercises) for $53 \%$ of the patients, and moderate compliance (that is, probably did the home exercises) for $33 \%$.

\section{PRIMARY OUTCOME MEASURES}

Table 2 presents follow up results for the primary outcome measures. At 24 weeks (12 weeks after completion of treatment), a beneficial effect was seen for pain during the past week. Compared with the post-treatment level (week 12) the effect size had declined to 0.36, indicating a small to moderate effect. No 
Table 2 Primary outcome measures: improvements and differences between intervention groups*

\begin{tabular}{|c|c|c|c|c|c|c|}
\hline & \multicolumn{2}{|c|}{ Exercise therapy } & \multicolumn{2}{|c|}{ Control } & \multirow[b]{2}{*}{$\begin{array}{l}\text { Difference }(95 \% \text { CI) } \\
\text { exercise therapy - controls }\end{array}$} & \multirow[b]{2}{*}{$\begin{array}{l}\text { Effec } \\
\text { size }\end{array}$} \\
\hline & $n$ & $\begin{array}{l}\text { Mean } \\
\text { changet }\end{array}$ & $n$ & $\begin{array}{l}\text { Mean } \\
\text { changet }\end{array}$ & & \\
\hline \multicolumn{7}{|c|}{ Pain past week } \\
\hline Week 12 & 93 & -22.8 & 98 & -5.7 & $-17.0(-23.6$ to -10.4$)$ & 0.58 \\
\hline Week 24 & 88 & -17.2 & 91 & -5.6 & $-11.5(-19.7$ to -3.3$)$ & 0.36 \\
\hline Week 36 & 90 & -15.5 & 92 & -8.9 & $-6.6(-14.7$ to 1.6$)$ & 0.20 \\
\hline \multicolumn{7}{|c|}{ Drug use: NSAIDs } \\
\hline Week 12 & 93 & $42 \%$ & 98 & $36 \%$ & $6 \%(-8 \%$ to $20 \%)$ & 0.12 \\
\hline Week 24 & 90 & $39 \%$ & 94 & $48 \%$ & $-9 \%(-22$ to $4 \%)$ & 0.18 \\
\hline Week 36 & 90 & $40 \%$ & 93 & $51 \%$ & $-10 \%(-23 \%$ to $3 \%)$ & 0.22 \\
\hline \multicolumn{7}{|c|}{ Observed disability } \\
\hline Week 12 & 91 & -0.21 & 98 & -0.02 & $-0.19(-0.38$ to -0.01$)$ & 0.28 \\
\hline Week 24 & 88 & -0.13 & 91 & -0.04 & $-0.09(-0.30$ to 0.12$)$ & 0.12 \\
\hline Week 36 & 88 & -0.16 & 91 & -0.06 & $-0.10(-0.31$ to 0.11$)$ & 0.13 \\
\hline
\end{tabular}

*Analyses are adjusted for baseline differences (fear avoidance beliefs towards physical activity, use of paracetamol, use of NSAIDs) and baseline score on specific outcome parameter.

†For drug use follow up scores are presented; percentage of patients taking drugs.

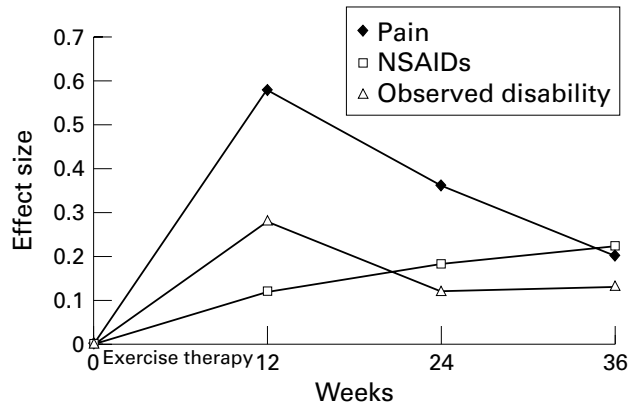

Figure 2 Effect sizes of primary outcome measures in the course of the trial.

effects were found for NSAIDs and observed disability. At 36 weeks (24 weeks after completing treatment), no differences were found between the intervention groups.

Figure 2 gives an overview of effect sizes over the course of the trial for the primary outcome measures. Beneficial effects for pain and disability were maximal at week 12 , just after completion of treatment. In the follow up period the size of the effects declined to non-significant levels in week 36 , except for the results for the use of NSAIDs, which remained stable during follow up.

SECONDARY OUTCOME MEASURES

Table 3 presents follow up results on the secondary outcome measures. At 24 weeks, beneficial effects of exercise were found for use of paracetamol and patient's global perceived effect. No effects were found for self reported disability, muscle strength, and range of motion. Similar effects were found at week 36 . As with the primary outcome measures, the size of the effects declined in the follow up period (week 12 to week 36). Again, an exception is found in the use of drugs where the effects of the use of paracetamol remained stable during follow up.

Exploratory analyses were performed to study the effects of exercise on muscle strength and joint range of motion only in patients with hip or knee OA. Two of 24 tests reached significance. At week 12 patients with knee OA in the group receiving exercise treatment showed improved muscle strength of the hip.
At week 24 patients with hip OA in the control group showed improved muscle strength of the hip.

OUTCOME IN PER-PROTOCOL ANALYSIS

A per-protocol analysis was conducted, excluding all patients who were classified as late ineligible patients owing to a changed medical diagnosis $(n=4)$ or owing to treatment not according to the protocol $(n=21)$. For 13 patients (eight of whom had been allocated to the exercise treatment group) treatment in the intervention period deviated from the protocol. Treatment in the follow up period had to be restricted to a minimum. However, in eight patients (five receiving exercise treatment and three controls) a medical specialist intervened, by performing a total hip replacement $(n=4)$, a total knee replacement $(n=1)$, an arthroscopy $(n=1)$, a meniscus operation $(n=1)$, or a corticosteroid injection $(\mathrm{n}=1)$.

The results of the per-protocol analysis were similar to the results of the intention to treat analysis. The effect sizes were somewhat higher, as can be explained by the nature of the analysis. This applies to both primary and secondary outcome measures (data not presented).

PROGNOSTIC VALUE OF PATIENT

CHARACTERISTICS FOR OUTCOME AFTER

COMPLETION OF SUPERVISED TREATMENT

Exploratory analyses were performed to study the prognostic value of demographic characteristics, clinical features, kinesiological characteristics, lifestyle, psychological wellbeing, and compliance for the residual effectiveness of exercise treatment. A study examined whether patients who were stable or improved in the follow up period (week 12 to week 36 ) differed from patients who deteriorated in this period, in their baseline characteristics or their compliance with treatment. Patients were classified as being stable/improved versus having deteriorated, based on their results for two primary outcome measures (pain during the past week and observed disability) after completion of treatment (week 12 to week 36) (see statistical analysis). A classification based on NSAIDs did not result in a useful division of patients (table 4).

Sixty two tests (31 patient characteristics and two outcome measures) were performed to study differences between patients who were stable/improved and those who had deteriorated. These patients groups differed in two aspects of their baseline characteristics. $\mathrm{Pa}-$ tients who were stable or showed improvement (in an observed disability) in the follow up period were more often overweight (body mass index $\geqslant 30)$ at baseline $(46 \% v 19 \%, \mathrm{p}=0.01)$. Patients whose pain was stable or improved in the follow up period reported lower levels on the pain coping strategy "transforming of pain" (8.3 $v 10.4, \mathrm{p}=0.001)$.

No significant differences were found for compliance in the supervised treatment period. Non-compliance, as reported by the patient, occurred as often in stable/improved patients as it occurred in patients who had deteriorated. 
Table 3 Secondary outcome measures: improvements and differences between intervention groups*

\begin{tabular}{|c|c|c|c|c|c|c|}
\hline & \multicolumn{2}{|c|}{ Exercise therapy } & \multicolumn{2}{|c|}{ Control } & \multirow{2}{*}{$\begin{array}{l}\text { Difference }(95 \% \text { CI) } \\
\text { exercise therapy - } \\
\text { controls }\end{array}$} & \multirow[b]{2}{*}{$\begin{array}{l}\text { Effect } \\
\text { size }\end{array}$} \\
\hline & $n$ & $\begin{array}{l}\text { Mean } \\
\text { changet }\end{array}$ & $n$ & $\begin{array}{l}\text { Mean } \\
\text { changet }\end{array}$ & & \\
\hline \multicolumn{7}{|c|}{ Drug use: paracetamol } \\
\hline Week 12 & 93 & $35 \%$ & 98 & $51 \%$ & $-16 \%(-29 \%$ to $-3 \%)$ & 0.33 \\
\hline Week 24 & 90 & $39 \%$ & 94 & $55 \%$ & $-17 \%(-30 \%$ to $-3 \%)$ & 0.32 \\
\hline Week 36 & 90 & $41 \%$ & 93 & $57 \%$ & $-17 \%(-30 \%$ to $-3 \%)$ & 0.32 \\
\hline \multicolumn{7}{|c|}{ Global perceived effect: improved } \\
\hline Week 12 & 91 & $47 \%$ & 98 & $18 \%$ & $28 \%(15 \%$ to $42 \%)$ & 0.64 \\
\hline Week 24 & 88 & $49 \%$ & 91 & $25 \%$ & $24 \%(10 \%$ to $38 \%)$ & 0.50 \\
\hline Week 36 & 90 & $46 \%$ & 93 & $29 \%$ & $17 \%(3 \%$ to $31 \%)$ & 0.35 \\
\hline \multicolumn{7}{|c|}{ Self reported disability $\S$} \\
\hline Week 12 & 93 & -1.1 & 98 & -0.0 & $-1.1(-2.3$ to 0.1$)$ & 0.26 \\
\hline Week 36 & 90 & $-1,2$ & 92 & $-0,3$ & $-0.8(-2.2$ to 0.5$)$ & 0.18 \\
\hline \multicolumn{7}{|c|}{ Muscle strength hip } \\
\hline Week 12 & 91 & 0.22 & 94 & 0.04 & $0.17(0.02$ to 0.33$)$ & 0.32 \\
\hline Week 24 & 85 & 0.16 & 87 & 0.13 & $0.04(-0.14$ to 0.21$)$ & 0.06 \\
\hline Week 36 & 85 & 0.26 & 87 & 0.22 & $0.04(-0.12$ to 0.21$)$ & 0.08 \\
\hline \multicolumn{7}{|c|}{ Muscle strength knee } \\
\hline Week 12 & 92 & 0.19 & 98 & 0.06 & $0.13(-0.04$ to 0.29$)$ & 0.22 \\
\hline Week 24 & 88 & 0.14 & 91 & 0.14 & $0.00(-0.17$ to 0.17$)$ & 0.00 \\
\hline Week 36 & 86 & 0.19 & 90 & 0.19 & $0.00(-0.18$ to 0.17$)$ & 0.01 \\
\hline \multicolumn{7}{|c|}{ Joint range of motion hip } \\
\hline Week 12 & 92 & 0.21 & 98 & 0.06 & $0.15(-0.03$ to 0.32$)$ & 0.23 \\
\hline Week 24 & 87 & 0.36 & 90 & 0.27 & $0.09(-0.13$ to 0.31$)$ & 0.11 \\
\hline Week 36 & 87 & 0.19 & 89 & 0.19 & $0.00(-0.21$ to 0.22$)$ & 0.01 \\
\hline \multicolumn{7}{|c|}{ Joint range of motion knee } \\
\hline Week 12 & 92 & 0.17 & 98 & 0.09 & $0.08(-0.09$ to 0.25$)$ & 0.13 \\
\hline Week 24 & 90 & 0.11 & 94 & 0.15 & $-0.04(-0.24$ to 0.17$)$ & 0.05 \\
\hline Week 36 & 87 & 0.11 & 90 & 0.05 & $0.05(-0.15$ to 0.25$)$ & 0.10 \\
\hline \multicolumn{7}{|c|}{ Physical activity: improved\$ } \\
\hline Week 12 & 87 & $46 \%$ & 97 & $48 \%$ & $-2 \%(-17 \%$ to $13 \%)$ & 0.04 \\
\hline Week 36 & 89 & $42 \%$ & 91 & $49 \%$ & $-8 \%(-22 \%$ to $7 \%)$ & 0.15 \\
\hline
\end{tabular}

*Analyses are adjusted for baseline differences (fear avoidance beliefs towards physical activity, use of paracetamol, use of NSAIDs) and baseline score on specific outcome parameter.

$\dagger$ For drug use follow up scores are presented; percentage of patients taking drugs.

$\ddagger$ Results on an eight point scale are dichotomised as improved (completely recovered, very much improved, and much improved) and not improved (slightly improved, not changed, slightly worsened, much worsened, and vastly worsened).

$\S$ Not assessed at 24 week follow up.

ๆResults are dichotomised as improved (increase in physical activity level, change $=1 \mathrm{~min} /$ week) versus not improved (stabilisation or decrease in physical activity, change $=0 \mathrm{~min} / \mathrm{week}$ )

Table 4 Residual effects in patients receiving exercise treatment: results at 36 weeks, compared with results at week $12(n=90)$

\begin{tabular}{llc}
\hline & Stable or improved patients ${ }^{\star}(n)$ & Patients who deteriorated $(n)$ \\
\hline Pain in past week & 46 & 44 \\
Drug use: NSAIDs & 84 & 6 \\
Observed disability† & 39 & 48 \\
\hline
\end{tabular}

${ }^{\star}$ Results are dichotomised as stable or improved (reduction or stabilisation of pain, drug use, or disability) versus deteriorated (increase of pain or disability, start taking NSAIDs, or increase in use).

†Missing values: 3

Table 5 Compliance with exercise treatment and its effect on the residual effect of exercise (results week 36 compared with week 12), as assessed by pain in the past week and observed disability

\begin{tabular}{llll}
\hline & Yes & No & p Value \\
\hline Compliance according to the patient (n) & 61 & 28 & \\
Pain in past week: stable or improved (\%) & 54.1 & 46.4 & 0.50 \\
Observed disability: stable or improved (\%) & 45.9 & 40.0 & 0.62 \\
Compliance according to the physiotherapist (n) & 73 & 11 & \\
Pain in past week: stable or improved (\%) & 56.2 & 27.3 & 0.07 \\
Observed disability: stable or improved (\%) & 46.6 & 22.2 & 0.17 \\
\hline
\end{tabular}

However, though not significant, noncompliance, as reported by the physiotherapist, seems to occur more often in patients who deteriorated during follow up than in stable/ improved patients (table 5).

\section{Discussion}

Several randomised clinical trials have shown the beneficial effects of exercise in patients with OA immediately after completion of treatment. ${ }^{9-14}$ Only a few, inconclusive studies have investigated whether effects are sustained over time. To rectify this lack of knowledge we studied the effectiveness of exercise in patients with OA at 12 and 24 weeks after completion of treatment. Our results indicate a slow decline of the beneficial effects of exercise treatment, resulting in a similar clinical status for patients receiving exercise treatment and controls.

Our results are in line with the few earlier studies on this topic, in the sense that all studies reported some decline in the post-treatment effects. ${ }^{9}{ }^{13-19}$ However, in most earlier work the decline seems less pronounced both in controlled trials ${ }^{913141617}$ and in one uncontrolled study. ${ }^{18}$ Several explanations are possible. The trials of Minor $^{14}$ and Deyle ${ }^{13}$ lacked an intention to treat analysis on follow up data. This probably resulted in an overestimation of treatment effects. ${ }^{41}$ In the FAST trial ${ }^{16}{ }^{17}$ treatment continued, albeit at home, until the last assessment. Therefore, no "non-training" effect could be studied. Interpretation of the results of Røgind et al is hampered by the high number of outcome measures, in combination with the small number of patients. ${ }^{9}$ In the uncontrolled study, ${ }^{18}$ four of 15 patients dropped out during the intervention. There, the remaining patients were probably a select and highly motivated group of patients, resulting in beneficial and sustaining effects.

Our results are in line with a prospective cohort study into the effects of stopping exercise programmes in healthy older adults. ${ }^{42}$ The beneficial effects on cardiovascular capacity and muscle strength after a 16 week cardiovascular or resistance exercise programme decreased after a 10 week non-training period.

Exploratory analyses were made on the prognostic value of patient characteristics for long term outcome. A beneficial long term outcome was found in patients who were overweight at baseline and in patients with a relatively low level of the specific pain coping strategy (transformation of pain). However, given the high number of tests performed $(n=62)$, it is entirely possible that these results are due to chance. A more thorough analysis of the factors affecting beneficial long term outcome is outside the scope of our study. However, future research should focus on these prognostic factors. A thorough analysis of the underlying mechanisms is needed, including analyses of interaction of these factors. We did not find a relation between compliance and beneficial outcome after completion of treatment. However, we did not have data on long term compliance - that is, on compliance after completion of the exercise treatment. Therefore, we have no insight into the highly relevant relation between long term compliance and outcome after completion of treatment. In future trials long term compliance should be studied.

The clinical implications of our results for the application of exercise treatment in patients with OA are clear. The evidence available clearly indicates the usefulness of exercise in patients with OA of the hip or knee. ${ }^{8-13}$ Small to moderate beneficial effects on pain and disability can be produced, which are the main symptoms of OA. However, the effects slowly 
decline, indicating that measures must be taken to maintain the beneficial effects of exercise. One measure is to introduce some form of retraining or prolonged training. Both intermittent exercise treatment and follow up booster sessions can be applied. There are some indications that the content and timing of an exercise programme affects results. In the FAST trial the relation between compliance and outcome was studied. ${ }^{43}$ The most beneficial outcome was found in patients with OA who did aerobic exercises three days a week for about 35 minutes each session. Patients spending more time exercising during a session had outcomes similar to those of non-exercising patients. These results are based on a secondary analysis of the dose-response relation between compliance and outcome. However, to our knowledge, no clinical studies on this topic are available as yet. The optimisation of content and timing of exercise treatment deserves further study.

Another measure is to maximise the patient's compliance with exercise, even after having completed a supervised programme. An important component of an exercise programme should be to facilitate acceptance and practice of exercises. Sluijs and Knibbe distinguished between short term and long term compliance and, subsequently, different approaches to be applied by healthcare providers. ${ }^{44}$ To improve long term compliance, a self regulated approach seems useful. In future trials, explicit attention should be paid to improvement of both short term and long term compliance.

In conclusion, exercise is effective in patients with OA of the knee or hip. However, these effects decline over time and finally disappear. Future research should focus on finding ways of optimally maintaining beneficial effects over time. Important issues are optimal content and timing of treatment and compliance of the patient with exercise therapy.

The authors thank the GPs, physiotherapists, and patients for participating in this study. We wish to express our gratitude to AH Hilvers, CR Visser, and GV Paalman for their contribution to the collection of the data.

Supported by a grant from the Dutch Fund of Investigative Medicine of the Dutch Health Insurance Council (OG92.066).

1 Hochberg MC, Altman RD, Brandt KD, Clark BM, Dieppe PA, Griffin MR, et al. Guidelines for the medical management of osteoarthritis. Part I. Osteoarthritis of the hip. Arthritis Rheum 1995;38:1535-40.

2 Hochberg MC, Altman RD, Brandt KD, Clark BM, Dieppe PA, Griffin MR, et al. Guidelines for the medical management of osteoarthritis. Part II. Osteoarthritis of the knee. Arthritis Rheum 1995;38:1541-6.

3 Altman RD, Hochberg MC, Moskowitz RW, Schnitzer TJ. Recommendations for the medical management of osteoarthritis of the hip and knee: 2000 update. Arthritis Rheum thritis of the hip and

4 Dieppe P. The classification and diagnosis of osteoarthritis. In: Kuettner KE, Goldberg VM, eds. Osteoarthritic disorders. Rosemont IL: American Academy of orthopaedic Surgeons, 1995:5-12.

5 Dekker J, Boot B, Woude LHV van der, Bijlsma JWJ. Pain and disability in osteoarthritis: a review of biobehaviora mechanisms. J Behav Med 1992;15:189-214.

6 Minor MA. Exercise in the management of osteoarthritis of the knee or hip. Arthritis Care Res 1994;7:198-204

7 Dekker J, Mulder PH, Bijlsma JWJ, Oostendorp RAB. Exercise therapy in patients with rheumatoid arthritis and osteoarthritis: a review. Advances in Behaviour Research and Therapy 1993;15:211-38.

8 Baar ME van, Assendelft WJJ, Dekker J, Oostendorp RAB, Bijlsma JWJ. Effectiveness of exercise therapy in patients with osteoarthritis of the hip or knee. A systematic review of randomized clinical trials. Arthritis Rheum 1999;42: 1361-9.
9 Røgind H, Bibow-Nielsen B, Jensen B, Møller HC, Frimodt-Møller H, Bliddal $\mathrm{H}$. The effects of a physical raining program on patients with osteoarthritis of the :1421-7.

10 O'Reilly SC, Muir KR, Doherty M. Effectiveness of home exercises on pain and disability from osteoarthritis of the knee: a randomised controlled trial. Ann Rheum Dis 1999; 58:15-19.

11 Maurer BT, Stern AG, Kinossian B, Cook, KD, Schumacher HR. Osteoarthritis of the knee: isokinetic quadriceps exercise versus an educational intervention. Arch Phys Med Rehab 1999;80:1293-9.

12 Kline Mangione K, McCully K, Gloviak A, Lefebvre I, Hofmann $M$, Craik R. The effects of high intensity and low-intensity cycle ergometry in older adults with knee osteoarthritis. J Gerontol 1999;54A:M184-90.

13 Deyle GD, Henderson NE, Mateketel RL, Ryder MG, Garber MB, Allison SC. Effectiveness of manual physical therapy and exercise in osteoarthritis of the knee. A randomized controlled trial. Ann Intern Med. 2000;132: 173-81.

14 Minor MA, Hewett JE, Webel RR, Anderson SK, Kay DR. Efficacy of physical conditioning exercise in patients with rheumatoid arthritis and osteoarthritis. Arthritis Rheum 1989;32:1396-405.

15 Sullivan T, Allegrante JP, Peterson, MGE, Kovar PA, MacKenzie CR. One-year followup of patients with osteoarthritis of the knee who participated in a program of supervised fitness walking and supportive patient education. Arthritis Care Res 1998;11:228-33.

16 Ettinger WH, Burns R, Messier SP, Applegate W, Rejeski WJ, Morgan T, et al. A randomized trial comparing aerobic exercise and resistance exercise with a health education program in older adults with knee osteoarthritis. JAMA $7 ; 277: 25-31$.

17 Messier SP, Thompson CD, Ettinger WH. Effects of long-term aerobic or weight training regimens on gait in an older, osteoarthritic population. J Appl Biomech 1997;13: 205-25.

18 Fisher NM, Pendergast DR, Gresham GE, Calkins E. Muscle rehabilitation: its effects on muscular and functional performance of patients with knee osteoarthritis. Arch Phys Med Rehab 1991;72:367-74

19 Marks R. Quadriceps exercises for osteoarthritis of the knee. A single case study comparing short-term versus long-term training effects. Physiotherapy 1994;80:195-9.

20 van Baar ME, Dekker J, Oostendorp RAB, Bijl D, Voorn ThB, Lemmens JAM, et al. The effectiveness of exercise therapy in patients with osteoarthritis of hip or knee: a randomised clinical trial. J Rheumatol 1998;25:2432-9.

21 Altman R, Asch E, Bloch D, Bole G, Borenstein D, Brandt $\mathrm{K}$, et al. Development of criteria for the classification and reporting of osteoarthritis: classification of osteoarthritis of the knee. Arthritis Rheum 1986;29:1039-49.

22 Altman R, Alarcón G, Appelrouth D, Bloch D, Borenstein $\mathrm{D}$, Brandt K, et al. The American College of Rheumatology criteria for the classification and reporting of osteoarthritis of the hip. Arthritis Rheum 1991;34:505-14

23 Altman RD, Fries JF, Bloch DA, Carstens J, Cooke TD, Genant $\mathrm{H}$, et al. Radiographic assessment of progression in osteoarthritis. Arthritis Rheum 1987;30:1214-25.

24 Cohen J. Statistical power analysis for the behavioral sciences. London: Academic Press, 1977.

25 Oostendorp RAB, van den Heuvel JH, Dekker J, van Baar ME. Exercise therapy in patients with osteoarthritis of knee or hip: a protocol. Amersfoort/Utrecht ,The Netherlands: NPI/ hip: a protocol.
NIVEL, 1998.

26 Keefe FJ, Caldwell DS, Queen K, Gil KM, Martinez S, Keefe FJ, Caldwell DS, Queen K, Gil KM, Martinez S,
Crisson JE, et al. Osteoarthritic knee pain: a behavioral analysis. Pain 1987;28:309-21.

27 Dekker J, Tola P, Aufdemkampe G, Winckers M. Categories of pain behaviour in osteoarthritis patients. Physiotherapy Theory and Practice 1993;9:157-63.

28 Keefe FJ. Block AR. Development of an observation method for assessing pain behaviour in chronic low back pain patients. Behaviour Therapy 1982;13:363-75.

29 McDaniel LK, Anderson, KO, Bradley LA, Young LD, Turner RA, Agudelo CA, et al. Development of an observation method for assessing pain behavior in rheumatoid arthritis. Pain 1986;24:165-84

30 van Baar ME, Dekker J, Lemmens JAM, Oostendorp RAB, Bijlsma JWJ. Pain and disability in patients with osteoarthritis of hip or knee: the relationship with articular, kinesiological and psychological characteristics. J Rheumatol 1998;25:125-33.

31 Steultjens MPM, Dekker J, van Baar ME, et al. Consistency and validity of an observational method for assessing disability in mobility in patients with osteoarthritis. Arthritis Care Res 1999;12:19-25.

32 van der Heijden GJMG. Shoulder disorder treatment. Efficacy of ultrasoundtherapy and electrotherapy. Maastricht, The Netherlands: University Press Maastricht, 1996.

33 Huiskes CJAE, Kraaimaat FW, Bijlsma JWJ. Development of a self-report questionnaire to assess the impact of rheumatic diseases on health and lifestyle. Journal of Rehabilitation Sciences 1990;3:65-70.

34 Bohannon RW. Muscle strength testing with hand-held dynamometers. In: Admundsen LR, ed. Muscle strength testing: instrumented and non-instrumented systems. New York: Churchill Livingstone, 1990:69-88.

35 Norkin CC, White DJ. Measurement of joint motion: a guide to goniometry. Philadelphia: FA Davis Co, 1986.

36 Caspersen CJ, Bloemberg BPM, Saris WHM, Merritt RK, Kromhout D. The prevalence of selected physical activities 
and their relation with coronary heart disease risk factors in elderly men: the Zutphen study, 1985. Am J Epidemiol $1991 ; 133: 1078-92$.

37 Altman R, Alarcón G, Appelrouth D, Bloch D, Borenstein $\mathrm{D}$, Brandt K, et al. The American College of Rheumatology criteria for the classification and reporting of osteoarthritis of the hand. Arthritis Rheum 1990;33:1601-10.

38 Passmore R, Eastwood MA. Human nutrition and dietetics. Edinburgh: Churchill Livingstone, 1986:270.

39 Kraaimaat FW, Bakker A, Evers AWM. Pijncoping strategieën bij chronische pijnpatiënten: De ontwikkeling van de Pijn-Coping-Inventarisatielijst (PCI). (Pain coping strategies in chronic pain patients: the development of the Pain Coping Inventory). Gedragstherapie 1997;30:185-201.

40 Waddell G, Newton M, Henderson I, Somerville D, Main CJ. A fear-avoidance beliefs questionnaire (FABQ) and the role of fear-avoidance beliefs in chronic low back pain and disability. Pain 1993;52:157-68.

41 Newell DJ. Intention-to-treat analysis: implications for quantitative and qualitative research. Int J Epidemiol 1992; 21:837-41.

42 Sforzo GA, McManis BG, Black D, Luniewski D, Scriber KC. Resilience to exercise detraining in healthy older aldults. J Am Geriatr Soc 1995;43:209-15.

43 Rejeski WJ, Brawley LR, Ettinger W, Morgan T, Thompson C. Compliance to exercise therapy in older participants with knee osteoarthritis: implications for treating disability. Med Sci Sports Exerc 1997;29:977-85.

44 Sluijs EM. Knibbe JJ. Patient compliance with exercises: different theoretical approaches to short-term and long-term compliance. Patient Educ Couns 1991;17:191-204.

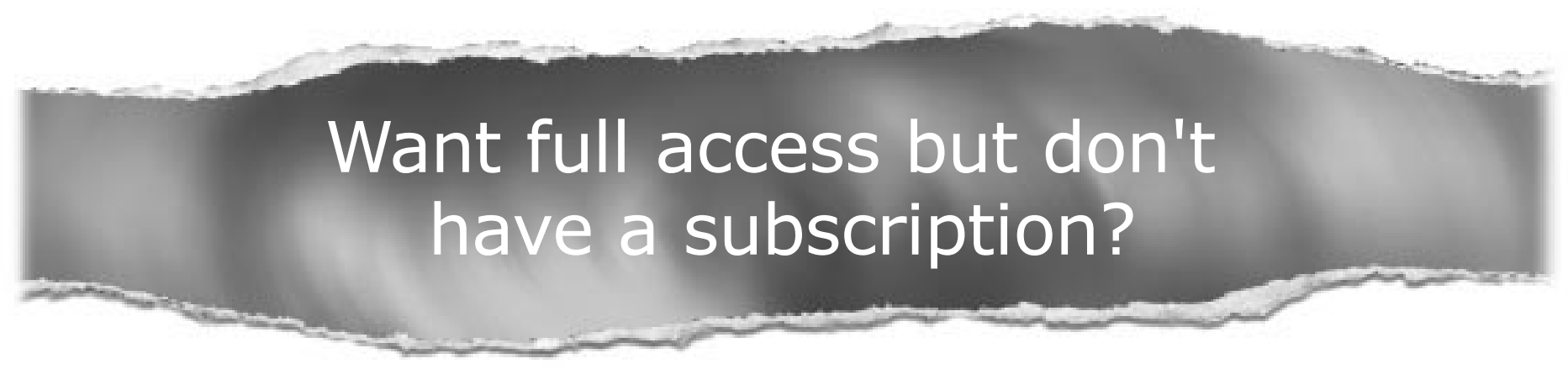

\section{Pay per access}

For just US $\$ 25$ you can have instant access to the whole website for 30 days. During this time you will be able to access the full text for all issues (including supplements) available. You will also be able to download and print any relevant pdf files for personal use, and take advantage of all the special features Annals of the Rheumatic Diseases online has to offer.

www.annrheumdis.com 